\title{
Carbohydrate of the Factor VIII/von Willebrand Factor in von Willebrand's Disease
}

\author{
T. S. Zimmerman, R. Voss, and T. S. Edgington, Department of Molecular \\ Immunology, Research Institute of Scripps Clinic, La Jolla, California 92037
}

\begin{abstract}
A B S T R A C T We have examined the plasma Factor VIII/von Willebrand factor (FVIII/vWF) molecule from 16 patients with von Willebrand's disease, and have found no evidence of a significant decrease of carbohydrate content in 15 of these patients. FVIII/vWF was isolated by preparative counter immunoelectrophoresis directly from plasma using antibody to Factor VIII-related antigen, reduced in sodium dodecyl sulfate in the presence of urea, and electrophoresed in $5 \%$ polyacrylamide gels to separate the FVIII/vWF subunit from other proteins. Duplicate gels were stained by either the periodic acid-Schiff (PAS) reaction or by Coomassie Brilliant Blue G250. The ratio of Coomassie:PAS was determined by spectrophotometric scanning of the gels. Transferrin was used as an internal reference standard. The ratio for 23 normal individuals was $2.4 \pm 0.38$ and the observed range was 1.8-3.8. 15 patients with von Willebrand's disease fell within this range. One patient independently reported as having decreased FVIII/vWF carbohydrate was also studied by this technique. A ratio of 6.8 was found, indicative of decreased, though not absent, carbohydrate. Cold insoluble globulin did not represent a significant contaminant in these analyses. 11 of the von Willebrand's disease patients with normal FVIII/vWF carbohydrate had abnormal crossed immunoelectrophoretic patterns characterized by absence of the less anodic forms of Factor VIII-related antigen. Four patients had normal patterns. These studies indicate that an absence or decrease of PAS reactive FVIII/vWF carbohydrate is not a consistent abnormality in von Willebrand's disease.
\end{abstract}

\section{INTRODUCTION}

A potential defect in glycosylation of Factor VIII/von Willebrand Factor (FVIII/vWF) ${ }^{1}$ in von Willebrand's

Portions of this material have been published in abstract form: 1978. Circulation. 58: 120. This publication 1812 from the Scripps Clinic \& Research Foundation.

Received for publication 21 May 1979 and in revised form 17 July 1979.

${ }^{1}$ Abbreviations used in this paper: CIEP, counterimmunoelectrophoresis; CIG, cold insoluble globulin; FVIII/ disease has been suggested by the studies of Gralnick and coworkers $(1,2)$. These investigators reported an absence of periodic acid-Schiff (PAS) reactive carbohydrate in the reduced FVIII/vWF subunit isolated from five different individuals with this disease. In two instances the sialic acid content of isolated FVIII/vWF preparations was analyzed and found to be decreased. This hypothesis has been supported by independent studies demonstrating that structural modification of the carbohydrate moiety of FVIII/vWF can alter ristocetin cofactor activity (3-5). To explore this issue we have developed a preparative counterimmunoelectrophoretic technique (CIEP) for direct isolation of FVIII/vWF from small amounts of plasma. Relative content of carbohydrate in the FVIII/vWF subunit was estimated by quantitative scanning of polyacrylamide gels stained with the PAS reaction. In one individual previously studied by Gralnick and coworkers $(1,2)$, PAS reactive carbohydrate was decreased. In 15 additional patients with this disease there was no significant difference from normal.

\section{METHODS}

CIEP isolation of FVIII/vWF. $23 \mathrm{ml}$ of $0.9 \%$ agarose (Sea Kem-ME Marine Colloids Division, FMC Corporation, Rockland, Maine) in $0.075 \mu \mathrm{m} \mathrm{B-2}$ barbital buffer (Beckman Instrument, Inc., Fullerton, Calif.) was layered on a $31 / 4 \times 4$-in. glass slide that had been precoated with agarose (6). A $1 \times 8-\mathrm{cm}$ antibody trough was cut and filled with the gamma globulin fraction of Factor VIII-related antigen (anti-VIII:Ag) antiserum that had been diluted in $0.9 \%$ agarose and maintained liquid at $56^{\circ} \mathrm{C}$. Anti-VIII:Ag antisera was prepared and absorbed as described (6). It was monospecific by crossed immunoelectrophoresis and did not form immunoprecipitates with either purified cold.insoluble globulin or fibrinogen on Ouchterlony analysis. The gamma globulin fraction was prepared by three cycles of precipitation at $50 \%$ saturation with ammonium sulfate then dialyzed against barbital buffer. The anti-VIII:Ag gamma globulin was dissolved in four parts agarose at $56^{\circ} \mathrm{C}$ to give a final concentration of 3 $\mathrm{mg} / \mathrm{ml}$. The agarose gamma globulin mixture was poured so

vWF, Factor VIII/von Willebrand factor; PAS, periodic acid-Schiff; vWd, von Willebrand's disease; VIII:Ag, Factor VIII-related antigen. 
as to fill the antibody trough and allowed to gel. An antigen well of $6 \times 2 \mathrm{~cm}$ was cut for 3-ml plasma samples or an $8 \times 2.5$ for 5-ml samples. The sample was then introduced, and the slide was electrophoresed at $15 \mathrm{~mA}$ for 2 to $3 \mathrm{~h}$, then at 7 $\mathrm{mA}$ for an additional $14 \mathrm{~h}$.

The single immunoprecipitate that formed was cut from the gel, placed in screw cap 15-ml polystyrene centrifuge tubes (Corning Glass Works, Science Products Div., Corning, N. Y.) which had been multiply perforated, and washed in two changes of 3.5 liters of $1.0 \mathrm{M}$ sodium chloride, $0.05 \mathrm{M}$ phosphate, $\mathrm{pH} 7.0$ buffer for $2-4 \mathrm{~d}$.

Polyacrylamide gel electrophoresis. The FVIII/vWF immunoprecipitates were then dialyzed against 2 liters of $0.06 \mathrm{M}$ Tris $\mathrm{HCl}, \mathrm{pH} 6.8$ for $4 \mathrm{~h}$, transferred to $13 \times 100-\mathrm{mm}$ glass tubes and heated in a boiling water bath until the agarose melted. Precipitates were combined as necessary to provide sufficient FVIII/vWF for satisfactory quantitative scanning of the sodium dodecyl sulfate polyacrylamide gels. The samples were lyophilized, then dissolved in $\cong 1 \mathrm{ml}$ of $5 \mathrm{M}$ urea, $1 \%$ sodium dodecyl sulfate, and $0.125 \mathrm{M}$ Tris hydrochloride, $\mathrm{pH} 6.8$ in a boiling water bath. The total volume was adjusted to $2.2 \mathrm{ml}$ with the same buffer and $30 \mu \mathrm{l}$ of dithiothreitol $(1.4 \mathrm{M})$ was added. The sample was boiled for 10 min and 1-ml aliquots were then transferred into separate tubes. Transferrin was then added and the mixture incubated at $45^{\circ} \mathrm{C}$ for $4 \mathrm{~h} .15 \mathrm{mg}$ of transferrin (Grade II, Sigma Chemical Co., St. Louis, Mo.) was added to all samples that were to be electrophoresed on gels subsequently stained with Coomassie Brilliant Blue G (Sigma Chemical Co.). $30 \mathrm{mg}$ of transferrin was added to all samples when the gels were to be stained with PAS. The samples were electrophoresed by the method of Laemmli (7) in $0.5 \mathrm{~cm}$ Diam polyacrylamide tube gels that contained $0.1 \%$ sodium dodecyl sulfate. The stacking gels were $7 \mathrm{~cm}$ and the running gels were $5 \mathrm{~cm}$ in length. Gels were stained with Coomassie Brilliant Blue $\mathrm{G}$ by the method of Reisner et al. (8) or with PAS by the method of Kapitany and Zebrowski (9).

Gel scanning. Gels were scanned using a Gilford automatic recording spectrophotometer model $2400-2$ outfitted with a Gilford gel scanner model 2520 (Gilford Instrument Laboratories Inc., Oberlin, Ohio). Absorbance was measured at $625 \mathrm{~nm}$ for the Coomassie-stained gels and at $542 \mathrm{~nm}$ for the PAS-stained gels. All gels were scanned at $5 \mathrm{~cm} / \mathrm{min}$ with a chart speed of $10 \mathrm{in} . / \mathrm{min}$. The calibrated absorbance range was usually 1.0 for PAS gels and 1.5 or 2 for Coomassiestained gels. Peak areas were multiplied by the calibrated absorbance used to allow gel-to-gel comparison. Each gel was scanned three times with $\cong 120^{\circ}$ rotation of axis between scans and the values averaged.

Calculations. The area of each peak was measured with a computing planimeter (Los Angeles Scientific Instrument Co., Inc., Los Angeles, Calif.). The mean area was calculated for the FVIII/vWF and transferrin bands on each gel. Gels run with transferrin only were included in each gel run. A small band (peak $I_{t}$ ) (Fig. 1) of the transferrin control gels overlapped the region of the FVIII/vWF peak on the FVIII/ vWF gels. The area attributable to the transferrin standard was subtracted from the area of the FVIII/vWF band. Correction for staining variations within a gel run were performed as follows:

FVIII/vWF corrected $=\left(\right.$ band $I-$ band $\left.I_{t}\right) \times \frac{\text { band } I_{t}}{\text { band II }}$.

The Coomassie:PAS ratio was calculated from the FVII/vWF area (corrected). Results of different runs were normalized by comparing the Coomassie:PAS ratio of band $\mathrm{II}_{t}$ of each run, to a "standard" transferrin Coomassie:PAS ratio. This standard

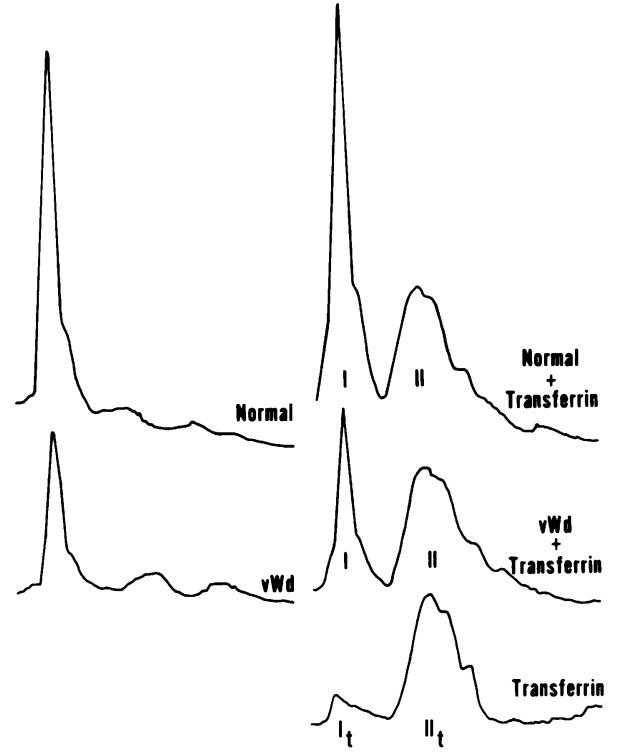

FIgURE 1 PAS stained SDS polyacrylamide gel scan tracings. Upper and lower left: CIEP precipitates of normal and $\mathrm{vWd}$ FVIII/vWF were reduced in SDS urea and electrophoresed in the absence of transferrin. Upper and middle right: Transferrin was added to the FVIII/vWF immunoprecipitates and the mixtures were electrophoresed. Lower right: Electrophoresis of transferrin alone. Peak $I$ is the FVIII/vWF peak. Peak II is the transferrin peak in gel runs in which transferrin was added to the FVIII/vWF immunoprecipitate. Peak $I_{t}$ is present when transferrin is run alone. This peak overlaps the FVIII/vWF peak. Peak II $_{t}$ is the transferrin peak when transferrin is run alone. The anode (bottom of the polyacrylamide gels) is to the right.

ratio was derived as the mean transferrin Coomassie:PAS ratio from 10 runs.

Plasma. $9 \mathrm{vol}$ of blood were collected into $1 \mathrm{vol}$ of $3.8 \%$ (wt/vol) trisodium citrate and centrifuged at 2,500 $\mathrm{g}$ for $15 \mathrm{~min}$ at either $4^{\circ}$ or $25^{\circ} \mathrm{C}$. Platelet-poor plasma thus obtained was then absorbed once with $5 \%$ aluminum hydroxide (Rehsorptar, Reheis Chemical Company, Phoenix, AZ) and three times with 2.5\% aluminum hydroxide. Aluminum hydroxide absorption did not effect the FVIII/vWF concentration in normal or von Willebrand's disease plasmas and the results were unchanged if absorption was omitted. Plasmas were obtained from: $(a)$ 23 normal donors. Levels of VIII:Ag in the normal plasma samples ranged from 0.45 to $2.1 \mathrm{U} / \mathrm{ml}$. (b) 16 patients with von Willebrand's disease (vWd) (Table I). These individuals had either plasma Factor VIII-related antigen concentration below $0.3 \mathrm{U} / \mathrm{ml}$ and normal crossed immunoelectrophoresis (type I) or abnormal crossed immunoelectrophoretic patterns with normal or decreased VIII:Ag concentrations (type II) (Table I). All had prolonged skin bleeding times.

Cryoprecipitation of plasma FVII/vWF was performed as described (10).

Column purified FVIII/vWF was prepared by agarose gel chromatography of cryoprecipitated FVIII/vWF as described (10). Column purified FVIII/vWF from four separate preparations was studied.

Radio-crossed immunoelectrophoresis was performed as described (11). VIII:Ag was measured by quantitative immunoelectrophoresis as described (6). $1 \mathrm{U}$ of VIII: Ag is equivalent to that present in $1 \mathrm{ml}$ of pooled plasma from 20 normal individuals. 
TABLE I

VIIIAg Concentration and Coomassie:PAS Ratios in vWd

\begin{tabular}{|c|c|c|}
\hline Patient & VIII:Ag & Coomassie:PAS ratio \\
\hline & $U / m l$ & \\
\hline \multicolumn{3}{|c|}{$\begin{array}{l}\text { Type I (normal crossed } \\
\text { immunoelectrophoresis) }\end{array}$} \\
\hline 2 & 0.13 & 2.9 \\
\hline 5 & 0.25 & 2.5 \\
\hline 6 & 0.15 & 3.0 \\
\hline 7 & 0.28 & 2.5 \\
\hline \multicolumn{3}{|c|}{$\begin{array}{l}\text { Type II (abnormal crossed } \\
\text { immunoelectrophoresis) }\end{array}$} \\
\hline 1 & 0.25 & 2.4 \\
\hline 3 & 0.60 & 2.1 \\
\hline 4 & 0.34 & 2.3 \\
\hline 7 & 0.28 & 2.5 \\
\hline 8 & 0.50 & 2.5 \\
\hline 9 & 0.48 & 2.4 \\
\hline 10 & 0.53 & 2.8 \\
\hline 11 & 1.34 & 2.4 \\
\hline 12 & 0.77 & 2.8 \\
\hline 13 & 0.18 & 2.6 \\
\hline 14 & 1.30 & 2.9 \\
\hline 15 & 0.18 & 2.3 \\
\hline 16 & 0.71 & 6.8 \\
\hline \multicolumn{3}{|c|}{ Normal individuals } \\
\hline$n=23$ & $\begin{array}{c}\text { mean } 1: 19 S D \pm 0.53 \\
\text { range, } 0.45-2.45\end{array}$ & $\begin{array}{l}2.4 \mathrm{SD} \pm 0.38 ; \\
\text { range, } 1.8-3.8\end{array}$ \\
\hline
\end{tabular}

Cold insoluble globulin (CIG) was purified by affinity chromatography according to the method of Engvall and Ruoslahti (12) and was kindly provided by Dr. Edward Plow, Scripps Clinic \& Research Foundation. It was labeled with ${ }^{125} \mathrm{I}$ by the chloramine $\mathrm{T}$ method (13), using a minimal limiting concentration of chloramine $\mathrm{T}$ consistent with iodination.

\section{RESULTS}

Isolation of FVIII/vWF by preparative CIEP. Recovery of FVIII/vWF by the CIEP technique was estimated in the following manner: Samples that contained 2-4 VIII:Ag U of column purified FVIII/vWF were placed in antigen wells and reisolated by CIEP. Immunoprecipitates were dissociated in sodium dodecyl sulfate and urea, reduced and electrophoresed on polyacrylamide gels with subsequent quantitation of the FVIII/vWF subunit band by spectrophotometric scanning. Recovery was compared with the same quantity of column purified FVIII/vWF directly analyzed on polyacrylamide gels. (Total absorbance of the FVIII/vWF band, derived from planimetric analysis of the spectrophotometric scan, was directly proportional to the quantity of FVIII/vWF applied to the gel whether quantitated for protein by Coomassie stain or for carbohydrate by PAS stain [Fig. 2].) Mean re- covery of FVIII/vWF by CIEP was $63 \%$ (range, 39$78 \% ; n=6)$.

The ratio of Coomassie Brilliant Blue G to PAS did not vary with the amount of FVIII/vWF applied to the gels if sufficient material was used so that the FVIII/vWF band peak area was $>1,400$ planimeter units when stained with Coomassie Brilliant Blue G or 700 planimeter $U$ when stained with PAS (Fig. 2). To achieve FVIII/vWF peaks that gave these values, $\cong 0.80 \mathrm{U}$ or more of VIII:Ag was applied. This required 1.5-3 $\mathrm{ml}$ of normal plasma for each gel. For cryosupernatant plasma and for vWd patients with VIII:Ag concentrations of between 0.13 and $0.15, \mathrm{U} / \mathrm{ml} \mathrm{CIEP}$ immunoprecipitates, each prepared from $5 \mathrm{ml}$ of plasma, were used for each gel.

The subunit of CIG has the same mobility on sodium dodecyl sulfate polyacrylamide gels as that of the FVIII/vWF subunit, therefore possible contamination of the FVIII/vWF band with CIG was assessed. Several lines of evidence indicate that no significant contamination was present. First, there is a good correlation between the VIII:Ag concentration of the starting plasma and FVIII/vWF subunit peak area on polyacrylamide gel scans (Fig. 3). Second, the Coomassie:PAS ratio of purified CIG was 7.4. This was significantly greater 


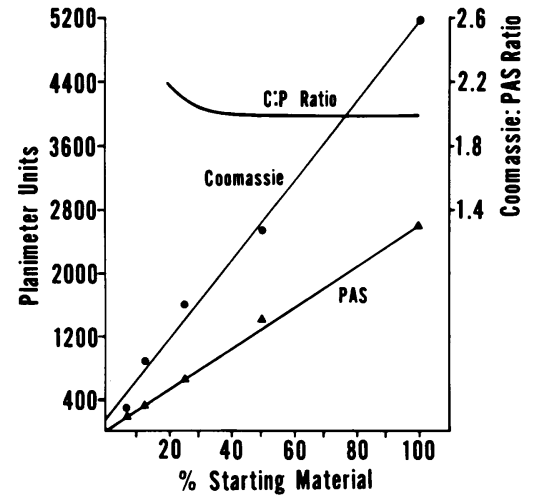

FIgURE 2 Calibration curve plotting relative immunoprecipitate concentration and area in planimeter units of the FVIII/vWF peak stained with either Coomassie Brilliant Blue $\mathrm{G}$ or PAS. The calculated least mean squares regression line is shown as is the Coomassie:PAS (C:P) ratio at different concentrations of the FVIII/vWF immunoprecipitate (upper line). Different dilutions of a typical CIEP immunoprecipitate isolated from $3 \mathrm{ml}$ of normal plasma were applied. $100 \%$ of starting material corresponds to the immunoprecipitate from $3 \mathrm{ml}$ of plasma, $50 \%$ of the starting material corresponds to the immunoprecipitate from $1.5 \mathrm{ml}$ of plasma and so on.

than the ratio of 2.4 for $\mathrm{FVIII} / \mathrm{vWF}$ purified by CIEP (mean of 23 samples from different normal individuals) or the ratio of 2.6 for the subunit from column purified FVIII/vWF (mean of four preparations from four different normal donors). Finally, ${ }^{125}$ I-CIG was added to starting plasma and FVIII/vWF then isolated by CIEP. After electrophoresis of the immunoprecipitate on polyacrylamide gels, the FVIII/vWF band was cut out and radioactivity determined. It was found that only $0.004 \%$ of the CIG added to plasma was present in the FVIII/vWF subunit band.

Precision of analysis. The Coomassie:PAS ratios were determined a total of 35 times on seven plasma

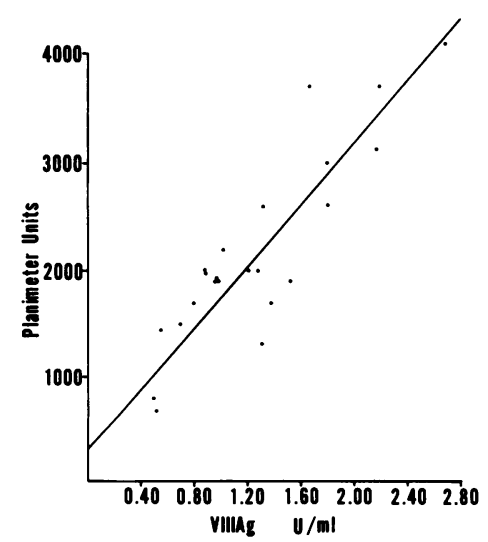

Figure 3 Correlation between FVIII/vWF peak area and VIII:Ag from 23 normal individuals. $3 \mathrm{ml}$ of plasma were used in the CIEP. The linear regression line of the points is indicated. The correlation coefficient was $0.89 ; P<0.01$. samples drawn from the same individual on different days. The mean for all determinations was $2.35 \pm 0.47$; range, 1.7-3.9; coefficient of variation, $21 \%$. In determining the Coomassie:PAS ratios of FVIII/vWF from normal and $\mathrm{vWd}$ individuals, two or three determinations were routinely made on each plasma sample from a given individual. Plasma samples were obtained only once from the same normal or vWd patients in most instances. However, the Coomassie:PAS ratio for one patient with $v W d$ type II was determined 16 times on four different plasma samples. The mean for all determinations was $2.3 \pm 0.81$; coefficient of variation, $35 \%$; range, $1.2-3.6$.

The possibility that the CIEP method was selectively isolating a restricted subset of the different FVIII/vWF forms was evaluated by comparing the Coomassie:PAS ratios of FVIII/vWF from cryosupernatant plasma (smaller, more anodic forms of FVIII/vWF) with that of highly purified FVIII/vWF from the void volume of the Sepharose CL-4B (Pharmacia Fine Chemicals, Piscataway, N. J.) column (the largest, least anodic forms of FVIII/vWF) and FVIII/vWF isolated by CIEP from the starting plasma from which the cryosupernate FVIII/vWF and the highly purified FVIII/vWF were obtained. In determinations on four separate samples of each, the mean Coomassie:PAS ratio of cryosupernatant FVIII/vWF was 2.7; for column purified FVIII/ vWF, 2.6; and for the starting plasma, 2.7.

The Coomassie:PAS ratios of normal individuals was $2.4, \mathrm{SD} \pm 0.38$, range, $1.8-3.8$. This variation is similar to that observed for multiple determinations of a single individual (see above) and therefore may only represent variability of the method. Even with this variability, a relative decrease of only $33 \%$ in reactive carbohydrate, relative to protein, would give a Coomassie:PAS ratio of 3.6 which is 3 SD beyond the mean of normal ratios.

15 of the 16 patients studied had Coomassie:PAS ratios within the normal range (Table I). There was no apparent difference between FVIII/vWF from individuals with vWd type I (decreased VIII:Ag with normal crossed immunoelectrophoresis) and vWd type II (abnormal crossed immunoelectrophoresis with absence of less anodic forms of VIII:Ag). One individual with vWd type II previously studied by Gralnick and coworkers $(1,2)$, had a clearly increased Coomassie:PAS ratio of 6.8 , range $5.9-7.4(n=3)$. This difference is significant at greater than the $99.9 \%$ level of confidence and reflects a $45 \%$ relative decrease in PAS reactive carbohydrate.

\section{DISCUSSION}

This study demonstrates that the PAS reactive carbohydrate content of plasma FVIII/vWF in vWd is usually indistinguishable from normal. However, it does con- 
firm a reduced carbohydrate content in one patient previously reported by Gralnick and coworkers $(1,2)$. This latter observation demonstrates that the CIEP technique combined with quantitative gel scanning is capable of detecting decreased FVIII/vWF carbohydrate in the infrequent cases where such an abnormality occurs.

The relative content of PAS reactive carbohydrate appears similar for the larger and smaller forms of FVIII/vWF from normal individuals. The larger forms have been shown to possess ristocetin cofactor activity, whereas the smaller forms have none (14). The single patient with reduced PAS reactive carbohydrate, as well as the majority of patients we studied, were of vWd type II, i.e., an abnormal crossed immunoelectrophoretic pattern with absence of the larger, less anodic forms of FVIII/vWF. This single patient excluded, we could demonstrate no difference in the PAS reactive carbohydrate between individuals with type II or type I vWd (low concentrations of Factor VIII but normal crossed immunoelectrophoresis).

There has been recent interest in the possible role of the carbohydrate moiety in mediating the von Willebrand factor function of FVIII/vWF, i.e., the ability of FVIII/vWF to support adhesion of platelets to the subendothelium and thus correct the bleeding time in vWd. It has been reported by Sodetz and coworkers (4) that removal of sialic acid reduces ristocetin cofactor activity of FVIII/vWF by $62 \%$. Though Gralnick (5) could not confirm this observation he is in agreement with Sodetz et al. (4) that subsequent oxidation or enzymatic removal of galactose residues essentially abolishes ristocetin cofactor activity of FVIII/vWF. Sodetz and coworkers (4) have also shown that asialo FVIII/vWF binds to the hepatic lectin - the receptor for galactose-and is rapidly removed from the circulation. These investigators propose that one type of vWd might result from either reduced sialylation during synthesis or an increased desialylation with subsequent rapid removal of FVIII/vWF from the circulation, presumably by binding to the hepatic receptor. The techniques used in this study do not address such a limited and selective modification of the FVIII/vWF carbohydrate. However, a reduction of sialic acid would decrease the net negative charge of FVIII/vWF making it less anodic on immunoelectrophoresis. The FVIII/vWF of all patients reported to date shows either a normal crossed immunoelectrophoresis pattern (type I) or a more anodic migration with absence of the less anodal forms of the Factor VIII (type II). These considerations as well as the studies reported here make it unlikely that a simple decreased carbohydrate content of FVIII/vWF is the underlying molecular defect in most cases of $\mathrm{vWd}$.

\section{ACKNOWLEDGMENTS}

We would like to thank Dr. Edward F. Plow for the gift of CIG, Doctors Dominique Meyer of Hopital de Bicetre, Kremlin Bicetre, France, Jan Over, University Hospital, Utrecht, The Netherlands, and Harvey Gralnick, National Institutes of Health, Bethesda, Md. for gifts of plasma samples, and Ms. Mary Gortmaker and Ms. Sharon Garland for preparation of the manuscript.

This study was supported by National Institutes of Health grants HL-15491, HL-16411, and HL-20517.

\section{REFERENCES}

1. Gralnick, H. R., B. S. Coller, and Y. Sultan. 1976. Carbohydrate deficiency of the factor VIII/von Willebrand factor protein in von Willebrand's disease. Science (Wash. D. C.). 192: $56-59$.

2. Gralnick, H. R., Y. Sultan, and B. S. Coller. 1977. von Willebrand's disease: combined qualitative and quantitative abnormalities. N. Engl. J. Med. 296: 1024-1030.

3. Sodetz, J. M., S. V. Pizzo, and P. A. McKee. 1977. Relationship of sialic acid to function and in vitro survival of human Factor VIII/von Willebrand Factor protein J. Biol. Chem. 252: 5538-5546.

4. Sodetz, J. M., J. C. Paulson, S. V. Pizzo, and P. A. McKee. 1978. J. Biol. Chem. 253: 7202-7206.

5. Gralnick, H. R. 1978. Factor VIII/von Willebrand Factor Protein. Galactose, a cryptic determinant of von Willebrand factor activity. J. Clin. Invest. 62: 496-499.

6. Zimmerman, T. S., L. W. Hoyer, L. Dickson, and T. S. Edgington. 1975. Determination of the von Willebrand's disease antigen (Factor VIII-related antigen) in plasma by quantitative immunoelectrophoresis. J. Lab. Clin. Med. 86: 152-159.

7. Laemmli, U. K. 1970. Cleavage of structural proteins during the assembly of the head of bacteriophage T4. Nature (Lond.). 227: 680-685.

8. Reisner, A. H., P. Nemes, and C. Bucholtz. 1974. The use of Coomassie brilliant blue G250 perchloric acid solution for staining in electrophoresis and isoelectric focusing on polyacrylamide gels. Anal. Biochem. 64: 509-516.

9. Kapitany, R. A., and E. J. Zebrowski. 1973. A high resolution PAS stain for polyacrylamide gel electrophoresis. Anal. Biochem. 56: 361-369.

10. Montgomery, R. R., and T. S. Zimmerman. 1978. von Willebrand's disease antigen II. A new plasma and platelet antigen deficient in severe von Willebrand's disease. J. Clin. Invest. 61: 148-1507.

11. Koutts, J., P. N. Walsh, E. F. Plow, J. W. Fenton, II, B. N. Bouma, and T. S. Zimmerman. 1978. Active release of human platelet Factor VIII-related antigen by adenosine diphosphate, collagen and thrombin.J. Clin. Invest. 62: 1255-1263.

12. Engvall, E., and E. Ruoslahti. 1977. Binding of soluble form of fibrinoblast surface protein fibronectin to collagen. Int. J. Cancer. 20: 1-5.

13. McConahey, P. J., and F. J. Dixon. 1966. A method of trace iodination of proteins for immunologic studies. Int. Arch. Allergy Appl. Immunol. 29: 185-189.

14. Over, J., B. N. Bouma, J. A. van Mourik, J. J. Sixma, R. Vlooswijk and I. Bakker-Woudenberg. 1978. Heterogeneity of factor VIII. I. Characterization of factor VIII present in the supernatant of cryoprecipitate. J. Lab. Clin. Med. 91: 32-34. 